\title{
QUALITATIVE MONITORING TO SUPPORT PRO-ACTIVE VO MANAGEMENT
}

\author{
Iris Karvonen, Martin Ollus, Mikko Uoti \\ VTT Industrial Systems, FINLAND \\ Iris.Karvonen@vtt.fi \\ Martin.Ollus@vtt.fi \\ Mikko.Uoti@vtt.fi
}

\begin{abstract}
The paper aims to contribute to the development of pro-active Virtual Organization (VO) management. A method called "VO qualitative monitoring" is proposed based on the analysis and monitoring of VO success factors. The approach is applicable especially for VOs which have abstract objectives (difficult to measure quantitatively) and require personal and organizational commitment. Also the first implementation of the method is described.
\end{abstract}

\section{INTRODUCTION}

Virtual Organizations (VO) are created to fulfill a specific task, for example a delivery of a product or service to a customer. The task of VO management is to support the achievement of the VO objectives, mainly the customer requirements in the defined time, cost and quality frame [Karvonen et al 2005]. To do this, the status of the VO should be identified and actions to correct or avoid deviations should be implemented. Decision about actions is usually based on the comparison of actual achieved behavior compared to the wanted behavior. Methods like task monitoring and VO performance measurement [Westphal 2007] and simulation [Hodik et al 2007] are developed to support this. The actions are supposed to be proactive in order to avoid also emerging deviations from the expected outcome. However, it is difficult to act proactively and to identify hidden problems which would cause deviations later.

There are different types of VOs and they require different approaches for the VO management. One of the main determining factors is the type of the VO objective [Karvonen et al 2005]. This paper proposes a method called "VO qualitative monitoring" tracing factors affecting to the VO performance but not visible in quantitative measurements. The approach is applicable especially for VOs which have abstract objectives, which are difficult to measure quantitatively and require personal and organizational commitment (chapter 2). The method is based on a qualitative VO partner success model presented in chapter 3. The paper also presents the first implementation of the methodology as a tool in chapter 4. Suggestions for further advancement of the methodology are presented in chapter 5. 


\section{DEVELOPMENT OF VO QUALITATIVE MONITORING}

\subsection{Definition \& objectives}

"VO qualitative monitoring" (VOQM) is defined here as the collection and analysis of qualitative aspects which affect the success or non-success of a VO. They do not typically have an immediate influence on the VO performance; the effects develop gradually and can be seen in the quantitative performance measurements in delay. The objective of VO qualitative monitoring is to support VO management by the identification of the VO status in a proactive manner. It may thus complement VO performance measurement. The qualitative factors include items like VO partner satisfaction, commitment and motivation which partly also describe "VO feelings" or "VO atmosphere". Periodical follow-up can support the identification of changes in the hidden factors or the "VO feelings".

\subsection{VO management approaches and VOQM}

Different VOs have different types of objectives, challenges and the operating environment. The main objective of a VO is to create value to the customer but most often constraints of time and costs exist. A VO, which has been able to create a high quality customer solution, but has exceeded the allowed costs or time, is usually not considered a success.

There are also differences with respect to the goal specificity, clarity and stability. Typically the customer requirements set up the expected outcome (product or service), but not all its details. In product development or innovation VOs the goal is necessarily not quite clear. Often the VO objective may change during its operation.

The objectives considered most important and most difficult affect the focus of the management. In case of a dynamic goal it is important to be able to guide the VO to understand and pursue the up-to-date goal. In some cases the main problem may be the technical integrability of the partners' results or contributions, whereas in some other cases most problems are encountered in keeping to the schedule and/or cost limit. If there is a shortage of resources their management within a specified frame of time and costs may become the main issue in a VO.

By definition a VO always has a common goal. The need for collaboration and mutual dependences between the partners may vary. The degree of dependency has effect on the intense of VO management. Though in typical business cases the role of VO management is very important, there may be some specific cases, where this role may, at least partly, be replaced by well defined rules and practices.

The objectives of a VO are necessarily not fully in line with the objectives of the VO participants. Partners are independent organizations and may participate in several VOs at the same time. The contribution and behavior of each partner in a VO is affected by its objectives. The objectives of the different coexistent VOs and the different organizations may cause conflicts, especially if there is a shortage of resources. Thus it is useful to consider the objectives and motivating factors of the participants. These are qualitative, often hidden factors. 
In the short term the companies want to achieve the customer satisfaction and make profit in their current VOs. For the long term, while working in a VO, they want to gain future potential (references, customers), learn \& increase their knowledge and / or test and improve the processes \& tools.

In [Karvonen et al. 2005] the VO descriptive parameters [Pedersen 1999] were analysed to identify needs for different VO management approaches. The main factors affecting the VO management approach were identified to be the type of VO objective, the importance of dependencies and risks (probability and consequences). The following (partly overlapping) management approaches were identified:

- Multi-organizational (collaborative) project management

- Encouragement approach

- Self-organizing approach

- Automatic control VOs

- Supply chain management approach.

VO qualitative monitoring is applicable especially for VOs which have abstract objectives (difficult to measure quantitatively) and require personal and organisational commitment, that is for the "Encouragement approach", most often also for Collaborative project management and potentially for the self-organizing approach.

VO qualitative monitoring aims to support the VO management. Thus it takes mostly the viewpoint of $\mathrm{VO}$ manager. It is planned to operate as a tool of VO manager or VO management team to monitor the qualitative status of the VO and thus to support the identification of potential deviations.

\subsection{Methodology for VOQM development}

As a basis to develop VOQM, a qualitative success model of factors affecting to VO partner performance has been used. The model has been presented as a success tree presenting the different factors required for the VO success. The tree format is able to present the logic (obligatory or alternative factors; and/or-gates) and enables the break down of the factors to lower level elements and factors. As an alternative, opposite logic, VO fault tree could be used.

For the VOQM, the success factors have been turned into a questionnaire. The same qualitative model has been used both for the "VO feelings" questionnaire where the VO partners assess the VO as a whole from their own viewpoint and for the "VO partner assessment" tool where the VO management team is planned to assess the VO partners. Each branch in the tree has been broken up to more detailed "leaves" and turned to questions. The questionnaire is presented in the form of statements. The idea is that the query participants assess the degree how far they agree or do not agree about the given statements.

Partly the statements deal with partner specific issues, partly they concern factors common to the whole project. In this way it is possible to collect views and opinions about the status of the whole VO, as reviewed from the VO partner. The collected information is aggregated together. The initial idea has not been that the VO manager is allowed to identify the single answers, even if this in some cases could be a workable method.

Even if the respondents are not identified, the information received with such a query cannot be considered completely reliable. The respondent may be willing to 
answer "as expected" or to hide some problems. The self-assessment may thus be partially distorted or even false. The higher the common trust in the network, the more reliable information can be expected.

\section{VO QUALITATIVE MONITORING CONTENTS}

\subsection{VO success model}

To build the needed base model the conditions of success were analysed: what is needed to achieve the VO success. The VO success is dependent on the performance of the VO partners and some common and external factors, like VO management actions and customer behavior. Figure 1 presents a high-level success tree presentation of $\mathrm{VO}$ partner performance. Here the external and common factors are also included in the VO partner model because they may prevent the partner to succeed in the VO; even if the partner itself may not be "guilty" to these deviations. In the success tree two kinds of "gates" are used:

- \&-gate (AND) means that the output (on the left side in this figure) is true only if all the inputs to the gate (on the right hand side) are true.

$\mathrm{V}$-gate (OR) means that the output is true if at least one of the inputs is true.

Thus the interpretation is that to perform as needed in a VO the partner must have (AND-gate) first the four elements:

- motivation (reason why to perform as required for the VO; especially in case of other concurrent and conflicting interests). The best alternative is that both the organization and the persons involved are motivated, in minimum not one of them prevents the other one from the work.

- $\quad$ knowledge/capability (professional skill)

- $\quad$ the possibility/ availability of resources (free resources / time).

- $\quad$ no failures or deviations occur

If one of these elements is missing the partner performance may fail. If motivation is not high enough, good results may not be achieved even if there is knowledge and time to perform the task. As well if the professional skill is not high enough, the results are not of high quality. If there is motivation and knowledge, but no time (free resources) to perform the task, it cannot be performed. Furthermore, even if all the other elements are in place, occasional failures may destroy the success.

Collaboration and mutual dependencies create additional challenges, which are here turned to requirements about understanding the $\mathrm{VO}$ goal, quality of VO management and collaboration.

Furthermore, each component can be analysed more in detail. For example, as described in figure 1, motivation may come from different sources (OR-gate):

expected benefit; in short or long term and potentially compared with competing benefits. A short-term benefit may be a good price received from the performance. Long-term benefits may be linked to the expectation of future contracts, development of customer relationships, increase of knowledge or support to the strategic objectives of the partner.

penalties or other threats in case of non-performance or 
- issues linked to human interaction, like values, will, duty, friendship, pleasure etc. These may be important if the motivation of a person/persons involved is important. This is especially the case in the areas where innovation is more important than routines. At the same time it must be noted, that the motivation of a person does not always lead to the motivation of the organization.

Similarly the other factors can be opened more to define in more detail, for example, what kind of knowledge and capabilities are needed, what are the prerequisites for sufficient resources etc.

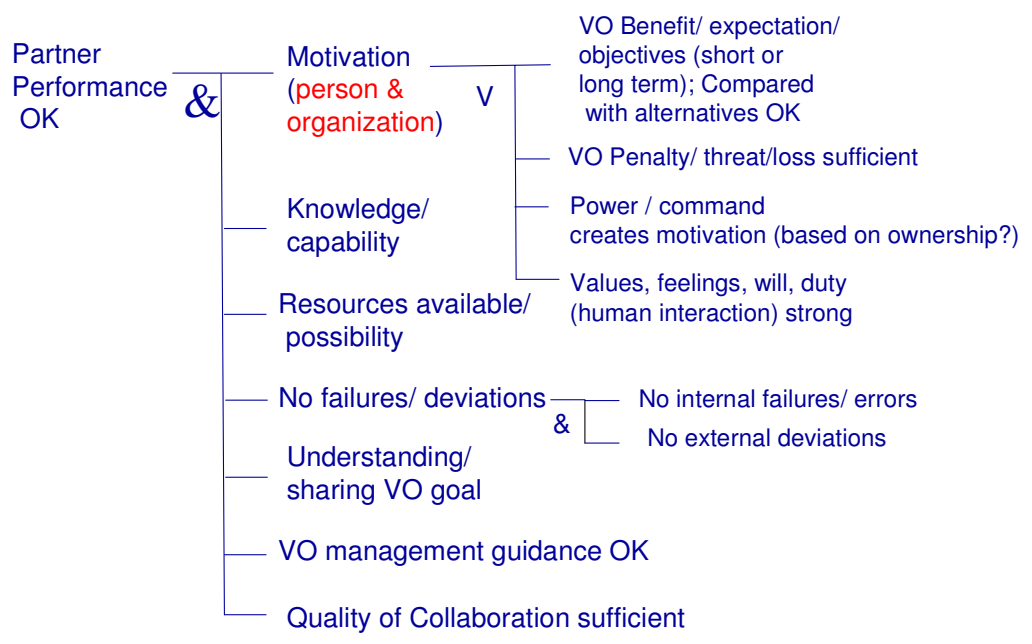

Figure 1. High level success tree for VO partner performance

\subsection{VO qualitative monitoring contents}

The success model presented above has been used as the base for developing the questionnaires for $\mathrm{VO}$ qualitative monitoring. The current version of $\mathrm{VO}$ qualitative monitoring tool and methodology includes two parts:

- identification of the VO qualitative status ("VO feelings" or "VO climate"), based on the views of all VO partners about their own status and the VO status as a whole - qualitative assessment of (selected or all) VO partners, planned to be based on the views of VO management team.

The current version of the partner questionnaire includes the following main topics, providing input to the mentioned issues:

- VO objectives \& results -> Is there a need to clarify the expected result?

- VO importance -> Measure of motivation of the person \& organization

- Knowledge\& resources -> Identify lack of knowledge/resources

- Collaboration -> Identify the status of collaboration

- VO/task performance-> identify problems

- VO management -> identify weaknesses in VO management

- Risk assessment- $>$ Free text on comments and proposals

If the query is performed repeatedly during the VO it can be able to reflect potential changes in the mentioned factors, like understanding, motivation and commitment. 
The above assessment is planned to be performed as a self-assessment: each partner or even each person participating the $\mathrm{VO}$ evaluates the presented factors. This assessment can be complemented with an assessment of VO partners performed by the VO manager or VO management team. The idea is that this partner assessment could be performed proactively already in the beginning of the $\mathrm{VO}$ to be able to foresee potential future problems, and then repeated during the VO operation as there is some experience about the running VO.

The assessment of partners uses the experience and opinions of VO manager and the management team. In principle it could collect views of all partners about all partners but this would in practise most often be too heavy. This assessment includes the criticality of the partner (defining how important the questions of the next topics are), commitment, performance and collaboration and comments. If the VO has a high number of partners it may not be useful to assess them all but select only the most critical ones for the analysis.

\subsection{Utilization for VO inheritance/ lessons learned}

VO inheritance is defined as "the practice of storing and passing on the experience and other non-proprietary assets created through collaboration in a VO. "[Karvonen et al. 2007]. The objective of VO inheritance is to enrich the VBE "bag of assets" [Afsarmanesh et al. 2008], thus improving the preparedness of the VBE for business opportunities. The added value, or the contents of the VO inheritance, is called "VO heritage".

The history of the "run-time" analysis of a VO could be saved and used as part of VO heritage. Even more benefit could be gained by comparing the received results later with the realized performance. This would require the comparison of the qualitative and quantitative results which is not necessarily straightforward.

Another way to support the inheritance is to create specific questionnaires performed at the VO dissolution phase. This analysis could be modified from the current questionnaires to collect views of the VO final success and experience.

\section{FIRST IMPLEMENTATION OF VOQM TOOL (VOF)}

VO qualitative monitoring is currently implemented as a web query to VO partners and VO management ("VOF"-tool). It allows an easy way of sharing and answering the questions by selecting the degree of agreeing/disagreeing of each statement. The results are aggregated without the identification of a single answer.

In the current version the number of statements is 41 for VO partner questionnaire (in 7 groups) and 13 (4 groups) for the VO management (for each partner). Additionally there are 6 free text questions for the partners and one free text comment for the VO management (for each partner).

The current "VOF" tool is implemented using a generic commercial web query tool [Digium 2008]. Respondents can be called to the query by e-mail offering a link to the query web site. The tool offers a selection of different summary alternatives, based on numbering the level of agreement (agree $=4$; somewhat agree $=3$; somewhat disagree $=2$, disagree $=1$ ). The statements have been formulated so that in each case disagreeing refers to a weakness. This enables the calculation of mean 
values for each question and further for each question group. Visualization and comparison of the mean values can support the identification of VO weaknesses.

The VO partners' assessment results may be aggregated and presented equally. Here the first question group assessing the criticality of the partner is different from those assessing their performance. It could be used to order the partners according to criticality. The creation of summaries is not as straightforward as different partners may get different number of assessments.

Sharing the analysis results is dependent on the network and the VO rules and practices. Typically the concluded results, not revealing the answers of any single organization or person, are accepted to be open within the VO. The results of the VO partner assessment, performed by the VO management, are necessarily not open outside the VO management.

The VOF tool has not been tested in industrial cases yet, but it has had a small experimentation in a special VO, ECOLEAD project. The invitation to the query was sent to all people working in the project. The results were partly used for the periodic reporting.

\section{CONCLUSIONS AND FURTHER DEVELOPMENT}

\subsection{Conclusions}

The paper presents an approach for the qualitative monitoring of a VO status, to enable the pro-active identification of potential emerging problems. The method with the first query has been implemented and preliminarily tested as a one-off analysis in one specific VO using a web query tool. The tool proved to be easy to take into use, it provided automatic summaries of the results and it was easy and light to use for the VO participants.

The first experimentation showed that the method can give interesting information about the VO status and the "feelings" of the VO partners which have an effect on the VO performance. The results obtained in the test looked reasonable. Even if it can be argued that the given information is not totally reliable, it can give impression about the attitude of the partners.

The experimentation also revealed needs and potential for further development of the methodology. One of the development possibilities lies in the area of adding dynamics in the methodology: Instead of one-off analysis it could be repeated regularly during the VO. This may require the creation of separate queries for the start, operation and dissolution phases. Further, the questionnaire base could be managed in the VBE or network and it could be developed as a learning tool, adding questions about new types of problem or disturbance sources, based on experience. It could also be possible to learn about the reliability of the assessments of the companies after deviations occurred - was this visible somehow in the qualitative assessment before the problem occurrence? Additional benefit can be expected by integrating the qualitative and quantitative (performance measurement) approaches and linking to VO model.

Finally, even if the current tool offers several options for the presentation of the results, new approaches for the visualization could be developed. These could include the visualization on the "VO dashboard" [Negretto et al. 2008] together with 
the quantitative measures or visualization as a success tree presenting the tree status with early alarms.

\subsection{Acknowledgments}

The paper is mainly based on work performed in the Integrated project ECOLEAD funded by the European Community under the Framework programme 6 (IP 506958).

\section{REFERENCES}

1. Afsarmanesh, H., Msanjila S., Ermilova, E. , Wiesner, S., Woelfel, W., Seifert, M. (2008). VBE Management System. In Camarinha-Matos L.M., Afsarmanesh H., Ollus M. (eds.) Methods and Tools for Collaborative Networked Organizations, Springer (to appear).

2. DIGIUM (2008). http://www.digium.fi/en/page313.html, April 2008.

2. Karvonen, I.; Salkari, I.; Ollus, M. (2005). Characterizing Virtual Organization and Their Management. In Camarinha-Matos, L., Afsarmanesh, H. \& Ortiz, A. (Ed.), Collaborative Networks and Their Breeding Environment (pp 193- 204). Springer.

3. Karvonen, I.,Salkari. I., Ollus, M. (2007) Identification of forms and components of VO inheritance. In Camarinha-Matos, L., Afrmanesh, H. , Novais, P. \& Analide, C., Establishing the Foundation of Collaborative Networks, Proc. 8th IFIP Working Conference on Virtual Enterprises, ( pp. 253262). Springer.

4. Negretto U, Hodík J, Král L, Mulder W, Ollus M, Pondrelli L, Westphal I. (2008). VO MANAGEMENT SOLUTIONS - VO Management e-Services. In: Camarinha-Matos L.M., Afsarmanesh H., Ollus M. (ecs.) Methods and Tools for Collaborative Networked Organizations, Springer (to appear).

5. Pedersen, J.D., Tolle, M. Vesterager, J. Final report on Models. Deliverable 1.3 of Esprit 26509 Globeman 21 project. 1999.

6. Westphal, I., Thoben,K. Seifert, M. (2007). Measuring Collaboration Performance in Virtual Organisations. In Camarinha-Matos, L., Afsarmanesh, H. , Novais, P. \& Analide, C., Establishing the Foundation of Collaborative Networks. Proc. 8th IFIP Working Conference on Virtual Enterprises (pp. 33-42). Springer.

7. Hodík, J., Mulder, W., Pondrelli, L., Westphal I. (2007) ICT services supporting virtual organization management. Proceedings of the 3rd I*PROMS Virtual International Conference, July 2-13, 2007. ISBN 978-1904445-52-4. 